\title{
Effect of lamination schemes on natural frequency and modal damping of fiber reinforced laminated beam using Ritz method
}

\author{
Somi Naidu Balireddy ${ }^{1,2}$ (D), Pitchaimani Jeyaraj ${ }^{1, *}$ (D), Lenin Babu Mailan Chinnapandi ${ }^{3}$ (D), and Ch V.S.N. Reddi ${ }^{4}$ \\ ${ }^{1}$ National Institute of Technology Karnataka Surathkal, Mangalore 575 025, India \\ ${ }^{2}$ Vignan's Institute of Information Technology, Visakhapatnam, Andhra Pradesh 530051, India \\ ${ }^{3}$ Vellore Institute of Technology Chennai, Tamilnadu 600 127, India \\ 4 Aditya Engineering College, Surampalem, Andhra Pradesh, India
}

Received: 8 January 2021 / Accepted: 6 August 2021

\begin{abstract}
The current study focussed on analysing natural frequency and damping of laminated composite beams (LCBs) by varying fiber angle, aspect ratio, material property and boundary conditions. Ritz method with displacement field based on the shear and normal deformable theory is used and the modal damping is calculated using modal strain energy method. Effects of symmetric angle-ply and cross-ply, anti symmetric cross-ply, balanced and quasi-isotropic lay up schemes on modal damping are presented for the first time. Results revealed that influence of lay-up scheme on natural frequencies is significant for the thin beams while the modal damping of the thin beams are not sensitive to lay-up scheme. However, the lay-up scheme influences the damping significantly for the thick beams. Similarly, high strength fiber reinforced LCBs have higher natural frequency while low strength fiber reinforced LCBs have higher damping due to the better fiber-matrix interaction.
\end{abstract}

Keywords: Ritz method / free vibration / damping / aspect ratio / LCB

\section{Introduction}

Fibre reinforced laminated structures are always in demand because of high strength and very less weight. The laminated composite beam is a very common structural element used in various engineering applications such as mechanical, automobile, marine and aircraft industries [1]. The structures made of laminated composite materials have higher damping compared to conventional metallic structures due to the filler-matrix interaction [2]. Rajesh and Jeyaraj [3] through experiments demonstrated that, for a fibre reinforced composite beam, modal damping is influenced by nature of reinforcement. Senthilkumar et al. [4] shown that fibre length and its content influences the natural frequency of LCB significantly.

Various theorems presented by several researchers to analyze the free vibration frequencies of LCBs, using numerical and analytical methods, are recently reviewed by Sayyad and Ghugal [5]. Vo et al. [6] presented a shear and normal deformation model to analyse natural frequencies of LCBs using Ritz method. Nguyen et al. [7] formulated a unified model to study the static and dynamic

\footnotetext{
* e-mail: bnaidus@gmail.com
}

behaviours of LCBs using Ritz method based on different theorems. Jeyaraj et al. [8] analysed sound radiation behaviour of a laminated composite plate using finite element method and found that increase in modal damping significantly reduces the vibration response at the resonances. Eltaher and Mohamed [9] studied stability characteristics of composite sandwich beams using differential quadrature method. Li et al. [10] used a unified higher order theorem based method to analyse natural frequencies of LCBs under the axial compression load. Nguyen et al. [11] presented an analytical model for the analysis of static and dynamic behaviours of LCBs using Ritz method.

Modal damping is capable of controlling vibration and sound levels when the system is excited at the resonant frequencies. Damping plays a vital role in the design of engineering components subjected to vibration and other dynamic loadings. Chandra et al. [12] presented a detailed study on damping of laminated composites and reported that modal strain energy method is used in general to estimate the damping theoretically. In aerospace applications, FRP composites are preferred due to higher inherent damping associated with it. The increase in inherent damping reduces the peak forced vibration responses significantly [13]. Ni and Adams [14] presented a method to 
estimate damping of a LCB considering bending-twisting coupling and compared the numerical results with the experiments. Recently, Ozer et al. [15] developed a finite element for the damping analysis of laminated composites. Different elastic constants are defined in the complex form in order to calculate the modal damping of the composite structure numerically. Li et al. [16] presented an energy based theoretical model to analyse the damping of thin FRP plate. Lin et al. [17] predicted modal damping of carbon and glass FRP plates using modal strain energy and finite element method. Bruyneel et al. [18] highlighted the importance of designing laminated composite structures with optimised parameters to withstand against the buckling load. Irhirane et al. [19] presented different failure modes associated with LCBs and concluded that still necessary studies needed to find the suitable failure criteria for the given LCB. Liao et al. [20] studied LCBs made of carbon-epoxy to analyse the interleaving effect on the damping behaviour.

Literature study revealed that damping analysis of LCBs is very important for its design considering vibration and other dynamic effects. Some researchers analysed vibration and damping behaviour of LCBs using numerical, analytical and experimental methods. However, there is no comprehensive study reported so far considering different types of lamination schemes, aspect ratio, boundary condition and material of the FRP on the combined vibration and damping behaviour of the LCBs. Hence, the effect of symmetric, un-symmetric, cross-ply, angle-ply, balanced-ply and quasi-isotropic lamination schemes on the vibration and damping of LCBs is analysed. The effect of aspect ratio, structural boundary conditions and type of FRP material (carbon-epoxy and glass-epoxy) on the natural frequency and damping of LCBs also investigated in this work. Numerical method formulated based shear and normal deformation theorem and Ritz method is used to obtain the natural frequencies while modal strain energy based method is used to obtain the modal damping of the LCBs.

\section{Methodology}

A LCB having length, width and thickness as L,b and h receptively as shown in Figure 1 is considered. The shear and normal deformation theorem is used to define the strain displacement relation [6] and is as follows

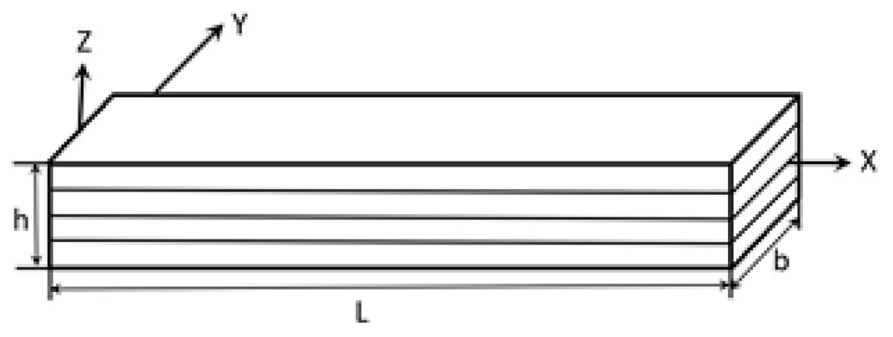

Fig. 1. Geometry of the analysed laminated beam.

$$
\begin{aligned}
U(x, z, t) & =u(x)-z \frac{\partial w_{b}(x, t)}{\partial x}-\frac{4 z^{3}}{3 h^{2}} \frac{\partial w_{s}(x, t)}{\partial x} \\
& =u(x, t)-z w_{b}^{\prime}(x, t)-f(z) w_{s}^{\prime}(x, t) \\
W(x, z, t) & =w_{b}(x, t)+w_{s}(x, t)+\left(1-\frac{4 z^{2}}{h^{2}}\right) \phi_{z}(x, t) \\
& =w_{b}(x, t)+w_{s}(x, t)+g(z) \phi_{z}(x, t)
\end{aligned}
$$

In the above equation $u, w_{b}, w_{s}$ and $\phi_{z}$ are the four unknown displacements of the mid-plane of the beam. The strains in axial, normal and shear are:

$$
\begin{gathered}
\varepsilon_{x}=\frac{\partial U}{\partial x}=u-z w_{b}^{\prime \prime}-f(z) w^{\prime \prime}{ }_{s} \\
\varepsilon_{z}=\frac{\partial W}{\partial z}=g^{\prime}\left(\phi_{z}\right) \\
\gamma_{x z}=\frac{\partial W}{\partial x}+\frac{\partial U}{\partial z}=g(z)\left(w_{s}^{\prime}+w^{\prime} \phi_{z}\right)
\end{gathered}
$$

For orthotropic lamina, the stress-strain relation is given by

$$
\left\{\begin{array}{l}
\sigma_{x} \\
\sigma_{y} \\
\sigma_{x z}
\end{array}\right\}=\left[\begin{array}{ccc}
Q_{11} & Q_{13} & 0 \\
Q_{13} & Q_{33} & 0 \\
0 & 0 & Q_{55}
\end{array}\right]^{k}\left\{\begin{array}{c}
\varepsilon_{x} \\
\varepsilon_{z} \\
\gamma_{x z}
\end{array}\right\}
$$

Strain energy variation $\partial U$ is given by:

See equation (4) below.

$$
\begin{aligned}
\partial U= & \int_{0}^{l} \int_{0}^{b}\left[\int_{\frac{-h}{2}}^{\frac{h}{2}}\left(\sigma_{x} \delta \varepsilon_{x}+\sigma_{x z} \delta \gamma_{x z}+\sigma_{z} g^{\prime} \delta \phi_{z}\right) d z\right] d y d x \\
= & \int_{0}^{l}\left[\left(A u^{\prime}-B w^{\prime \prime}{ }_{s}+X \phi_{z}\right) \delta u^{\prime}-\left(B u^{\prime}-D w^{\prime \prime}{ }_{b}-D_{s} w^{\prime \prime}{ }_{s}+Y \phi_{z}\right) \delta w^{\prime \prime}{ }_{b}\right. \\
& -\left(B_{s} u^{\prime}-D_{s} w^{\prime \prime}{ }_{b}-H w^{\prime \prime}{ }_{s}+Y_{s} \phi_{z}\right) \delta w^{\prime \prime}{ }_{s}+A_{s}\left(w^{\prime}{ }_{s}+\phi^{\prime}{ }_{z}\right)\left(\delta w^{\prime}{ }_{s}+\delta \phi^{\prime}{ }_{z}\right) \\
& \left.+\left(X u^{\prime}-Y w^{\prime \prime}{ }_{b}-Y_{s} w^{\prime \prime}{ }_{s}+Z \phi_{z}\right)\right] d x
\end{aligned}
$$


Table 1. Comparison of natural frequencies calculated using present method with Nguyen et al. [11] results.

\begin{tabular}{|c|c|c|c|c|c|}
\hline Aspect ratio $(L / h)$ & $\mathrm{BC}$ & Lay-up & Nguyen et al. [11] & Present study & $\%$ error \\
\hline 5 & & & 8.609 & 8.580 & 0.33 \\
\hline 10 & SS & $0^{\circ} / 90^{\circ} / 0^{\circ}$ & 18.814 & 18.847 & 0.17 \\
\hline 50 & & & 30.859 & 30.936 & 0.25 \\
\hline 5 & & & 1.234 & 1.184 & 4.00 \\
\hline 10 & $\mathrm{CF}$ & $0^{\circ} / 90^{\circ}$ & 1.322 & 1.234 & 6.60 \\
\hline 50 & & & 1.353 & 1.325 & 2.00 \\
\hline
\end{tabular}

variation in the kinetic energy $\delta k$ of the beam is,

\section{See equation (5) below.}

The equation of equilibrium are obtained by following the Hamilton's principle. The present study used Ritz procedure in finding the solution. The displacement functions for variables $u(x), w_{b}(x), w_{s}(x)$ and $w_{z}(x)$ are given as [1],

$$
u(x)=\sum_{j=1}^{m} A_{j} \Theta_{j}(x), \Theta_{j}(x)=\left(x+\frac{L}{2}\right)^{p_{u}}\left(x-\frac{L}{2}\right)^{q_{u}} x^{j-1}
$$

$$
w_{b}(x)=\sum_{j=1}^{m} B_{j} \varphi_{j}(x), \varphi_{j}(x)=\left(x+\frac{L}{2}\right)^{p_{w_{b}}}\left(x-\frac{L}{2}\right)^{q_{w_{b}}} x^{j-1}
$$

$$
w_{s}(x)=\sum_{j=1}^{m} C_{j} \zeta_{j}(x), \zeta_{j}(x)=\left(x-\frac{L}{2}\right)^{p_{w_{s}}}\left(x-\frac{L}{2}\right)^{q_{w_{s}}} x^{j-1}
$$

$$
w_{z}(x)=\sum_{j=1}^{m} D_{j} \psi_{j}(x), \psi_{j}(x)=\left(x+\frac{L}{2}\right)^{p_{w_{z}}}\left(x-\frac{L}{2}\right)^{q_{w_{z}}} x^{j-1}
$$

The terms $A_{j}, B_{j}, C_{j}$ and $D_{j}$ are undetermined coefficients, $\theta_{j}(x), \varphi(x), \zeta_{j}(x)$ and $\psi_{j}(x)$ are trial functions. The coefficients such as $p_{u}, q_{u}, p_{w b}, q_{w b}, p_{w s}, q_{w s}$ and $q_{w z}$ vary according to the end conditions of the LCB analysed. Substitution of the displacement functions in the equilibrium equations leads to the following typical eigenvalue problem which is used to calculate the natural frequencies of the LCB's studied.

$$
\left(K-\omega_{k}^{2} M\right) \phi_{k}=0
$$

In the above equation, $K$ and $M$ are stiffness and mass matrices respectively. $\omega_{k}$ is the natural frequency and $\phi_{k}$ is the mode shape [2]. It should be noted that the elastic properties of the material used in the present work are of complex in nature. Hence, the stiffness matrix evaluated also will be of complex in nature i.e., it consists of real and imaginary parts represented as $K_{I}$ and $K_{R}$ respectively.

Based on the modal strain energy method, the modal loss factor $\left(\eta_{k}\right)$ of $k$ th mode is obtained. as follows,

$$
\eta_{k}=\frac{\phi_{k}^{T} K_{I} \phi_{k}}{\phi_{k}^{T} K_{R} \phi_{k}}
$$

In the above equation, $\phi_{k}$ is the $k$ th mode shape and $\mathrm{K}_{I}$ is the imaginary part of the stiffness matrix. The reader is referred to reference [11] for more details regarding the relations stiffness and mass matrix co-efficients.

\section{Validation study}

\subsection{Natural frequency validation}

LCB beam analysed by Nguyen et al. [11] is considered for the validation of natural frequency calculation using the present study. Both Nguyen et al. [11] and present methods used same theorem and Ritz method to evaluate the nondimensional fundamental frequency. The results of the both the studies shown good agreement as shown in Table 1.

$$
\bar{\omega}=\frac{\omega L^{2}}{h} \sqrt{\frac{\rho}{E_{1}}}
$$

$$
\begin{aligned}
K= & -\int_{0}^{l} \int_{0}^{b}\left[\int_{\frac{-h}{2}}^{\frac{h}{2}} \rho(\dot{U} \delta \dot{U}+\dot{W} \delta \dot{W}) d z\right] d y d x \\
= & \int_{0}^{l}\left[\delta \dot{u}\left(m_{0} \dot{u}-m_{1} \dot{w}_{b}^{\prime}-m_{f} \dot{w}_{s}^{\prime}\right)+\delta \dot{w}_{b}\left[m_{0}\left(\dot{w}_{b}+\dot{w}_{s}\right)+m_{g} \dot{\phi}_{z}\right]\right. \\
& +\delta \dot{w}_{b}^{\prime}\left(-m_{1} \dot{u}+m_{2} \dot{w}_{b}^{\prime}+m_{f z} \dot{w}_{s}^{\prime}\right)+\delta \dot{w}_{s}\left[m_{0}\left(\dot{w}_{b}+\dot{w}_{s}\right)+m_{g} \dot{\phi}_{z}\right] \\
& \left.+\delta \dot{w}_{s}^{\prime}\left(-m_{f} \dot{u}+m_{f z} \dot{w}_{b}^{\prime}+m_{f^{2}} \dot{w}_{s}^{\prime}\right)+\delta \dot{\phi}_{z}\left[m_{g}\left(\dot{w}_{b}+\dot{w}_{s}\right)+m_{g^{2}} \dot{\phi}_{z}\right]\right] d x
\end{aligned}
$$


Table 2. Material properties of composite beams used in the study [17].

\begin{tabular}{lll}
\hline Material properties & Glass epoxy & Carbon epoxy \\
\hline$E_{1}(\mathrm{GPa})$ & 37.78 & 172.70 \\
$E_{2}(\mathrm{GPa})$ & 10.90 & 7.20 \\
$G_{12}(\mathrm{GPa})$ & 4.91 & 3.76 \\
$\nu_{12}(\mathrm{GPa})$ & 0.30 & 0.30 \\
$\rho \frac{\mathrm{kg}}{\mathrm{m}^{3}}$ & 1870 & 1566 \\
$\eta_{1}$ & 0.87 & 0.45 \\
$\eta_{2}$ & 5.05 & 4.22 \\
$\eta_{12}$ & 6.91 & 7.05 \\
\hline
\end{tabular}

\section{Results}

The study intended to predict the effect of boundary conditions, aspect ratio and various types of laminate schemes on natural frequency and damping of glass epoxy and carbon epoxy LCBs. The beams are investigated for SS, CC and CF boundary conditions. Symmetric angle-ply and cross-ply, anti-symmetric cross-ply, balanced and quasi-isotropic laminates are also analysed additionally. A LCB having a cross section of $b \times h$ and of length $(L)$ $0.5 \mathrm{~m}$ is considered in the study. It is assumed that the cross section is square and thickness is varied with respect to the given aspect ratio $(L / h)$. In order to analyse thick and thin beam cases, the LCB is investigated for two aspect ratios $(L / h=5$ and 20). The material properties of both glass epoxy (GE) and carbon epoxy HM-S (CE) used in the study are presented in Table 2 [17]. For both the materials, resin DX-210 is used. An increment of $15^{\circ}$ for $\theta$ is considered for the symmetric $\left(0^{\circ} / \theta^{\circ} / 0^{\circ}\right)$ laminates and corresponding variation in natural frequency and damping values for GE-LCB are presented in Tables 3 and 4 for the two different $L / h$ ratios. The LCBs are analysed for their first three bending modes. A comprehensive study on widely used laminate schemes for natural frequency and damping of fundamental mode is presented in Tables 5 and 6 for the two $L / h$ ratios respectively. Symmetric angle-ply and cross-ply, anti-symmetric cross-ply, balanced and quasiisotropic laminates are analysed for their first fundamental mode. The considered laminated schemes are analysed for both glass epoxy and carbon epoxy materials.

Table 3 is tabulated for natural frequency and damping of symmetric $\left(0^{\circ} / \theta^{\circ} / 0^{\circ}\right) \mathrm{LCB}$ with an aspect ratio of 5 . The results clearly depicts effect of boundary condition is more influential than effect of fiber angle. The depiction is due to, stiffness of the beam is directly influenced to the change in boundary condition. CC beams are more stiffer than other two beams and hence these are having higher natural frequency as anticipated. The variation in the values of natural frequency and damping observes to be marginal along the fiber angle variation. But, the frequency amplitude of $\mathrm{CC}$ beams are starting from high frequency band and that of SS and CF beams are falling in between lower frequency band and medium frequency band. Modal damping of SS-LCB modes are much higher than the other LCBs under other two boundary conditions due to the less structural stiffness.

The variation in natural frequency and modal damping of symmetric $\left(0^{\circ} / \theta^{\circ} / 0^{\circ}\right) \mathrm{LCB}$ with $L / h=20$ is presented in Table 4. Unlike the LCB with $L / h=5$, there is marginal variation in natural frequency for all the boundary conditions. However, there is significant change in the damping values as observed for $L / h=5$ cases. It is also observed that due to the higher aspect ratio the LCBs with $L / h=20$ have lower damping values compared to the corresponding LCBs with $L / h=5$, except for few cases.

Effect of lamination scheme, boundary condition and aspect ratio on the GE-LCB and CE-LCB is reported in Tables 5 and 6 , respectively. From the results of Table 5 it is clear that there is a greater variation in natural frequencies and damping values with regard to GE-LCB and CE-LCB. In general, natural frequencies of CE-LCB are higher than that of the GE-LCB due to higher elastic modulus associated with $\mathrm{CE}$ material. However, the modal damping values of GE-LCBs are much higher than the CE-LCBs due to the better fibre-matrix interaction, this can be clearly observed for thick beams. The fibermatrix interaction is better in GE-LCBs as the relatively weaker glass fiber able to interact more with the the matrix compared to the strong carbon fiber. In the case of lay-up scheme, higher natural frequency is obtained for CE-LCBs with symmetric cros-ply $\left(0^{\circ} / 90^{\circ} / 90^{\circ} / 0^{\circ}\right)$ for both the thin and thick beams. Similarly higher damping is observed for GE-LCB with anti-symmetric angle-ply $\left(45^{\circ} /-45^{\circ} / 45^{\circ} /-45^{\circ}\right)$.

\section{Conclusion}

Free vibration and damping investigation on glass epoxy and carbon epoxy LCBs with various configurations including boundary conditions, aspect ratio, fiber angle and lay up is presented. The solutions in the study are obtained using Ritz method with polynomial displacement field. From the results the following conclusions are drawn:

- The type of boundary conditions significantly effecting the natural frequency and damping of LCBs.

- Natural frequencies of thick beams are not much sensitive to lamination scheme while, damping of thick beams is highly sensitive to the lamination scheme.

- Modal damping of thin beams are not much influenced by the lamination scheme while the natural frequency of thin beams is very much sensitive to lamination scheme.

- Natural frequencies and modal damping are also highly sensitive to the nature of the reinforced material. The relatively strong fiber reinforcement enhances natural frequencies due to the higher elastic modulus associated with it. The relatively weak fiber reinforcement enhances modal damping due to the better fibermatrix interaction. 
Table 3. Natural frequencies and modal damping of $0^{\circ} / \theta^{\circ} / 0^{\circ}$ GE-LCB beam with $L / h=5$.

\begin{tabular}{|c|c|c|c|c|}
\hline \multirow{2}{*}{$\begin{array}{l}\text { Fiber angle } \\
0^{\circ} / \theta^{\circ} / 0^{\circ}\end{array}$} & \multirow[t]{2}{*}{ Mode } & \multicolumn{3}{|c|}{ Boundary condition } \\
\hline & & SS & $\mathrm{CC}$ & $\mathrm{CF}$ \\
\hline \multirow{3}{*}{$0^{\circ}$} & 1st Mode & $264.96(0.3735)$ & $1727.92(0.0103)$ & $290.73(0.0078)$ \\
\hline & 2nd Mode & $592.99(0.2499)$ & $4380.23(0.0108)$ & $1686.37(0.0085)$ \\
\hline & 3rd Mode & $791.59(0.0249)$ & $4616.32(0.0055)$ & $2308.16(0.0055)$ \\
\hline \multirow{3}{*}{$15^{\circ}$} & 1st Mode & $264.49(0.3750)$ & $1725.64(0.0103)$ & $290.28(0.0078)$ \\
\hline & 2nd Mode & $592.04(0.2515)$ & $4375.10(0.0108)$ & $1684.00(0.0085)$ \\
\hline & 3rd Mode & $790.47(0.0248)$ & $4549.21(0.0059)$ & $2274.60(0.0059)$ \\
\hline \multirow{3}{*}{$30^{\circ}$} & 1st Mode & $263.50(0.3780)$ & $1720.19(0.0103)$ & $289.21(0.0079)$ \\
\hline & 2nd Mode & $590.24(0.2552)$ & $4362.72(0.0109)$ & $1678.34(0.0086)$ \\
\hline & 3rd Mode & $787.78(0.0246)$ & $4383.46(0.0069)$ & $2191.73(0.0069)$ \\
\hline \multirow{3}{*}{$45^{\circ}$} & 1st Mode & $262.85(0.3800)$ & $1714.56(0.0104)$ & $288.13(0.0079)$ \\
\hline & 2nd Mode & $589.68(0.2584)$ & $4202.98(0.0079)$ & $1672.58(0.0086)$ \\
\hline & 3rd Mode & $785.06(0.0242)$ & $4349.84(0.0109)$ & $2101.49(0.0079)$ \\
\hline \multirow{3}{*}{$60^{\circ}$} & 1st Mode & $263.05(0.3794)$ & $1711.08(0.0103)$ & $287.49(0.0078)$ \\
\hline & 2nd Mode & $591.37(0.2592)$ & $4081.83(0.0083)$ & $1669.11(0.0086)$ \\
\hline & 3rd Mode & $783.45(0.0235)$ & $4341.69(0.0108)$ & $2040.92(0.0083)$ \\
\hline \multirow{3}{*}{$75^{\circ}$} & 1st Mode & $263.75(0.3772)$ & $1709.89(0.0102)$ & $287.29(0.0077)$ \\
\hline & 2nd Mode & $594.06(0.2581)$ & $4033.95(0.0082)$ & $1668.01(0.0085)$ \\
\hline & 3rd Mode & $782.97(0.0230)$ & $4338.75(0.0108)$ & $2016.97(0.0082)$ \\
\hline \multirow{3}{*}{$90^{\circ}$} & 1st Mode & $264.13(0.3760)$ & $1709.74(0.0102)$ & $287.28(0.0077)$ \\
\hline & 2nd Mode & $595.34(0.2574)$ & $4025.37(0.0080)$ & $1667.92(0.0085)$ \\
\hline & 3rd Mode & $782.94(0.0228)$ & $4338.31(0.0108)$ & $2012.69(0.0080)$ \\
\hline
\end{tabular}

Table 4. Natural frequencies and modal damping of $0^{\circ} / \theta^{\circ} / 0^{\circ}$ GE-LCB beam with $L / h=20$.

\begin{tabular}{lllll}
\hline Fiber angle & Mode & \multicolumn{3}{c}{ Boundary Condition } \\
\cline { 2 - 4 } $0^{\circ} / \theta^{\circ} / 0^{\circ}$ & & CC & CF \\
\hline \multirow{3}{*}{$0^{\circ}$} & 1st Mode & $206.98(0.0062)$ & $468.24(0.0072)$ & $73.64(0.0068)$ \\
& 2nd Mode & $475.24(0.5420)$ & $1280.84(0.0114)$ & $459.91(0.0067)$ \\
& 3rd Mode & $829.35(0.0068)$ & $2578.07(0.3069)$ & $1280.95(0.0068)$ \\
$15^{\circ}$ & 1st Mode & $206.65(0.0062)$ & $467.80(0.0074)$ & $73.52(0.0068)$ \\
& 2nd Mode & $475.33(0.5420)$ & $1441.58(0.1089)$ & $459.19(0.0068)$ \\
& 3rd Mode & $828.04(0.0068)$ & $2295.85(0.0059)$ & $1278.93(0.0068)$ \\
$30^{\circ}$ & 1st Mode & $205.87(0.0063)$ & $466.25(0.0074)$ & $73.25(0.0069)$ \\
& 2nd Mode & $476.03(0.5409)$ & $1278.94(0.0071)$ & $457.48(0.0069)$ \\
& 3rd Mode & $824.88(0.0069)$ & $2220.04(0.0084)$ & $1274.16(0.0069)$ \\
$45^{\circ}$ & 1st Mode & $205.09(0.0064)$ & $464.35(0.0074)$ & $72.97(0.0069)$ \\
& 2nd Mode & $477.97(0.5372)$ & $1273.95(0.0072)$ & $455.75(0.0069)$ \\
& 3rd Mode & $821.61(0.0069)$ & $2255.72(0.0076)$ & $1269.34(0.0069)$ \\
$60^{\circ}$ & 1st Mode & $204.62(0.0064)$ & $463.25(0.0074)$ & $72.81(0.0069)$ \\
& 2nd Mode & $481.13(0.5309)$ & $1271.06(0.0071)$ & $454.73(0.0068)$ \\
& 3rd Mode & $819.55(0.0069)$ & $2267.71(0.0072)$ & $1266.51(0.0069)$ \\
$75^{\circ}$ & 1st Mode & $204.48(0.0063)$ & $462.91(0.0072)$ & $72.76(0.0068)$ \\
& 2nd Mode & $484.19(0.5247)$ & $1270.16(0.0070)$ & $454.44(0.0068)$ \\
& 3rd Mode & $818.82(0.0068)$ & $2272.56(0.0070)$ & $1265.68(0.0068)$ \\
$90^{\circ}$ & 1st Mode & $204.47(0.0063)$ & $462.88(0.0072)$ & $72.76(0.0068)$ \\
& 2nd Mode & $485.47(0.5222)$ & $1270.09(0.007)$ & $454.42(0.0067)$ \\
& 3rd Mode & $818.71(0.0068)$ & $2273.61(0.007)$ & $1265.63(0.0067)$ \\
\hline
\end{tabular}


Table 5. Influence of lamination scheme on frequency and modal damping of the fundamental mode of LCB with $L / h=5$.

\begin{tabular}{llllll}
\hline Laminate type & Lay up & Material & SS & CC & CF \\
\hline Symmetric angle-ply & $30^{\circ} /-45^{\circ} /-45^{\circ} / 30^{\circ}$ & GE & $258.83(0.4122)$ & $1455.75(0.0141)$ & $240.83(0.0128)$ \\
& & CE & $353.47(0.1872)$ & $2613.57(0.0137)$ & $489.81(0.0050)$ \\
Symmetric cross-ply & $0^{\circ} / 90^{\circ} / 90^{\circ} / 0^{\circ}$ & GE & $263.13(0.3831)$ & $1663.94(0.0101)$ & $278.6(0.0077)$ \\
& & CE & $383.81(0.1523)$ & $3052.7(0.0166)$ & $613.17(0.0046)$ \\
Anti-Symmetric angle-ply & $45^{\circ} /-45^{\circ} / 45^{\circ} /-45^{\circ}$ & GE & $263.92(0.4174)$ & $1251.02(0.0189)$ & $205.01(0.0183)$ \\
& & CE & $387.24(0.1593)$ & $2046.65(0.0115)$ & $358.58(0.0066)$ \\
Anti-Symmetric cross-ply & $0^{\circ} / 90^{\circ} / 0^{\circ} / 90^{\circ}$ & GE & $281.26(0.3474)$ & $1380.51(0.013)$ & $228.23(0.0116)$ \\
& & CE & $468.94(0.0996)$ & $2385.83(0.0129)$ & $438.65(0.0054)$ \\
Balanced Laminate & $45^{\circ} / 30^{\circ} /-30^{\circ} /-45^{\circ}$ & GE & $266.73(0.3984)$ & $1282.10(0.0179)$ & $210.54(0.0172)$ \\
& & CE & $398.79(0.1407)$ & $2138.18(0.0119)$ & $380.24(0.0062)$ \\
Quasi Iso-tropic & $0^{\circ} / 45^{\circ} /-45^{\circ} / 90^{\circ}$ & GE & $278.99(0.3565)$ & $1293.77(0.0164)$ & $212.97(0.0155)$ \\
Quasi Iso-tropic & & CE & $465.14(0.1017)$ & $1927.61(0.0143)$ & $334.58(0.0110)$ \\
& $0^{\circ} / 60^{\circ} /-60^{\circ}$ & GE & $271.83(0.3740)$ & $1318.9(0.0171)$ & $217.28(0.0162)$ \\
& & CE & $426.17(0.1235)$ & $1908.21(0.0155)$ & $329.46(0.0129)$ \\
\hline
\end{tabular}

Table 6. Influence of lamination scheme on frequency and modal damping of the fundamental mode of LCB with $L / h=20$.

\begin{tabular}{llllll}
\hline Laminate type & Lay up & Material & SS & CC & CF \\
\hline Symmetric angle-ply & $30^{\circ} /-45^{\circ} /-45^{\circ} / 30^{\circ}$ & GE & $171.01(0.0119)$ & $280.83(0.1081)$ & $60.89(0.0122)$ \\
& & CE & $353.41(0.0038)$ & $798.32(0.0039)$ & $125.97(0.0037)$ \\
Symmetric cross-ply & $0^{\circ} / 90^{\circ} / 90^{\circ} / 0^{\circ}$ & GE & $198.17(0.0067)$ & $448.61(0.0073)$ & $70.54(0.0069)$ \\
& & CE & $447.39(0.0026)$ & $1009.06(0.0029)$ & $159.53(0.0026)$ \\
Anti-symmetric angle-ply & $45^{\circ} /-45^{\circ} / 45^{\circ} /-45^{\circ}$ & GE & $145.37(0.0179)$ & $329.46(0.0182)$ & $51.79(0.0180)$ \\
& & CE & $256.24(0.0060)$ & $204.59(0.3743)$ & $91.34(0.0060)$ \\
Anti-Symmetric cross-ply & $0^{\circ} / 90^{\circ} / 0^{\circ} / 90^{\circ}$ & GE & $162.03(0.0113)$ & $366.91(0.0114)$ & $57.74(0.0113)$ \\
& & CE & $315.68(0.0044)$ & $713.81(0.0045)$ & $112.60(0.0043)$ \\
Balanced laminate & $45^{\circ} / 30^{\circ} /-30^{\circ} /-45^{\circ}$ & GE & $149.34(0.0168)$ & $331.06(0.0106)$ & $53.20(0.0169)$ \\
& & CE & $272.26(0.0054)$ & $525.89(0.0313)$ & $97.05(0.0054)$ \\
Quasi-isotropic & $0^{\circ} / 45^{\circ} /-45^{\circ} / 90^{\circ}$ & GE & $151.16(0.0152)$ & $342.43(0.0153)$ & $53.88(0.0152)$ \\
& & CE & $239.28(0.0105)$ & $542.13(0.0105)$ & $85.43(0.0105)$ \\
Quasi-isotropic & $0^{\circ} / 60^{\circ} /-60^{\circ}$ & GE & $154.28(0.0158)$ & $349.47(0.0159)$ & $54.99(0.0158)$ \\
& & CE & $235.58(0.0125)$ & $533.91(0.0125)$ & $84.14(0.0125)$ \\
\hline
\end{tabular}

\section{References}

1. A. Karamanli, M. Aydogdu, Buckling of laminated composite and sandwich beams due to axially varying in-plane loads, Compos. Struct. 210, 391-408 (2019)

2. M.P. Arunkumar, M. Jagadeesh, J. Pitchaimani, K.V. Gangadharan, M.L. Babu, Sound radiation and transmission loss characteristics of a honeycomb sandwich panel with composite facings: effect of inherent material damping, J. Sound Vibr. 383, 221-232 (2016)

3. M. Rajesh, J. Pitchaimani, Experimental investigation on buckling and free vibration behavior of woven natural fiber fabric composite under axial compression, Compos. Struct. 163, 302-311 (2017)
4. K.S. Kumar, I. Siva, P. Jeyaraj, J.W. Jappes, S.C. Amico, N. Rajini, Synergy of fiber length and content on free vibration and damping behavior of natural fiber reinforced polyester composite beams, Mater. Des. (1980-2015) 56,379-386 (2014)

5. A.S. Sayyad, Y.M. Ghugal, Bending, buckling and free vibration of laminated composite and sandwich beams: a critical review of literature, Compos. Struct. 171, 486-504 (2017)

6. T.P. Vo, H.T. Thai, M. Aydogdu, Free vibration of axially loaded composite beams using a four-unknown shear and normal deformation theory, Compos. Struct. 178, 406-414 (2017)

7. T.K. Nguyen, B.D. Nguyen, T.P. Vo, H.T. Thai, A novel unified model for laminated composite beams, Compos. Struct. 238, 111943 (2020) 
8. P. Jeyaraj, N. Ganesan, C. Padmanabhan, Vibration and acoustic response of a composite plate with inherent material damping in a thermal environment, J. Sound Vibr. 320, 322-338 (2009)

9. M.A. Eltaher, S.A. Mohamed, Buckling and stability analysis of sandwich beams subjected to varying axial loads, Steel Compos. Struct. 34, 241-260 (2020)

10. J. Li, X. Hu, X. Li, Free vibration analyses of axially loaded laminated composite beams using a unified higher-order shear deformation theory and dynamic stiffness method, Compos. Struct. 158, 308-322 (2016)

11. N.D. Nguyen, T.K. Nguyen, T.P. Vo, H.T. Thai, Ritz-based analytical solutions for bending, buckling and vibration behavior of laminated composite beams, Int. J. Struct. Stab. Dyn. 18, 1850130 (2018)

12. R. Chandra, S.P. Singh, K. Gupta, Damping studies in fiberreinforced composites - a review, Compos. Struct. 46, 41-51 (1999)

13. P. Jeyaraj, N. Ganesan, C. Padmanabhan, Vibration and acoustic response of a composite plate with inherent material damping in a thermal environment, J. Sound Vib. 320, 322-338 (2009)

14. R.G. Ni, R.D. Adams, The damping and dynamic moduli of symmetric laminated composite beams - theoretical and experimental results, J. Compos. Mater. 18, 104-121 (1984)
15. M.S. Ozer, H. Koruk, K.Y. Sanliturk, Development of an equivalent shell finite element for modelling damped multilayered composite structures, Compos. Struct. 254, 112828 (2020)

16. H. Li, Y. Niu, Z. Li, Z. Xu, Q. Han, Modeling of amplitudedependent damping characteristics of fiber reinforced composite thin plate, Appl. Math. Model. 80, 394-407 (2020)

17. D.X. Lin, R.G. Ni, R.D. Adams, Prediction and measurement of the vibrational damping parameters of carbon and glass fiber-reinforced plastics plates, J. Compos. Mater. 18, 132-152 (1984)

18. M. Bruyneel, B. Colson, P. Jetteur, C. Raick, A. Remouchamps, S. Grihon, Recent progress in the optimal design of composite structures: industrial solution procedures on case studies, Int. J. Simul. Multidiscipl. Des. Optim. 2, 283-288 (2008)

19. E.H. Irhirane, J. Echaabi, M. Hattabi, M. Aboussaleh, A. Saouab, A comparative study of failure criteria applied to composite materials, Int. J. Simul. Multidiscipl. Des. Optim. 2, 141-147 (2008)

20. F.S. Liao, A.C. Su, T.C. Hsu, Vibration damping of interleaved carbon fiber-epoxy composite beams, J. Compos. Mater. 28, 1840-1854 (1994)

Cite this article as: Somi Naidu Balireddy, Pitchaimani Jeyaraj, Lenin Babu Mailan Chinnapandi, Ch V.S.N. Reddi, Effect of lamination schemes on natural frequency and modal damping of fiber reinforced laminated beam using Ritz method, Int. J. Simul. Multidisci. Des. Optim. 12, 15 (2021) 